\title{
Capacity Improvement in Spread Spectrum Watermarking using Biorthogonal Wavelet
}

\author{
Santi P. Maity \\ E \& TC Engg. Dept. \\ Bengal Engg. \& Sc. University \\ Shibpur, Howrah - 711 103, India \\ spmaity@telecom.becs.ac.in
}

\author{
Malay K. Kundu \\ Machine Intelligence Unit \\ Indian Statistical Institute \\ Kolkata - 700 108, India \\ malay@isical.ac.in
}

\author{
Mrinal Kr. Mandal \\ Elec. \& Comp. Engg. Dept. \\ University of Alberta \\ Edmonton,Canada T6G2V4 \\ mandal@ece.ualberta.ca
}

\begin{abstract}
Spread spectrum (SS) modulation is used widely to design robust digital watermarking schemes but in most cases due importance is not given on capacity and data imperceptibility aspects. In this paper, properties of spreading code are studied and a robust SS watermarking scheme is designed accordingly to embed multiple messages in different directions after signal decomposition. It has been observed that biorthogonal wavelet decomposition offers higher embedding capacity due to better directional selectivity compared to classical dyadic wavelet transform. Successive interference cancelation (SIC) is applied to improve robustness performance significantly compared to matched filter detection.
\end{abstract}

Index Terms-Biorthogonal wavelet, embedding capacity, SIC, SS watermarking.

\section{INTRODUCTION}

Several digital watermarking techniques, based on spread spectrum (SS) modulation principle, have recently been proposed. These techniques are typically used in transform domains such as DCT [1], Fourier-Mellin [2], and wavelet [3]. SS is accomplished by spreading narrow band watermark signal over many frequency bins of the cover image using pseudo random spreading sequences so that the watermark energy content for each bin becomes small and could hardly be detected. At the same time, any attempt to remove watermark causes image impairment to an extent that fails to preserve the acceptable quality of the watermarked image. This robustness attribute of SS watermarking scheme lies to a great extent on the properties of the code pattern. Apart from robustness criterion, two other essential requirements of digital watermarking scheme are data imperceptibility and high embedding capacity. A major limitation of the SS watermarking techniques is that large bandwidth requirement does not facilitate the extraction of a long bit sequence (payload requirement). In addition, any residual correlation between the host signal and watermark can result an unreliable detection.

In this paper, we propose an efficient SS watermarking technique to address the above limitations. The proposed technique uses biorthogonal wavelet to decompose an image in different directions (channels) and multiple watermark messages are then embedded successively in the subbands. Successive interference cancelation (SIC) is used in the proposed technique to improve robustness and embedding capacity. Experimental results have shown that embedding capacity can be improved three to four times with almost no loss of data imperceptibility and with marginal degradation in robustness performance.

\section{REVIEW OF THE BACKGROUND WORK}

SS image watermarking reported in [1]-[3] allows high embedding capacity but non blind detection process reduces their practicability in real life scenario. On the other hand, the majority of the reported blind SS technique uses a pseudo random signature as watermark and a binary decision is made whether the digital media is marked or unmarked by calculating the correlation between the watermark and the media under consideration. However pseudo random signature like watermark does not represent any meaningful information about the owner, user or media itself and thus serves limited application of watermarking. These algorithms can be modified for multiple bit embedding, but the 
stability of the decision variable demands fulfilment of some properties of the code patterns eg. they should be distinct, typically have vary small average value, orthogonal to each other (or close to it), and uncorrelated with the host signal [4]. In [4] Maity et al proposed DWT based digital image watermarking where Walsh-Hadamard $(\mathrm{W}-\mathrm{H})$ matrix is used to modulate pseudo noise (PN) code pattern for improvement in zero averaging and orthogonality properties. It is also suggested that data should be embedded in LL and HH subbands for better spectrum spreading. In this paper, we have investigated how data embedding capacity as well as robustness can be enhanced keeping imperceptibility of the hidden data unaltered.

\section{IMPROVEMENT IN EMBEDDING CAPACITY}

In case of normal DWT decomposition, if the embedding rate becomes high, data imperceptibility becomes lower and robustness performance is also decreased. Interference may occur as different sets of spreading codes (used for different watermark messages) are added with the decomposed cover image signal using single scaling function. Moreover, the decomposition does not always yield low correlation with the code patterns and high robustness may not be achieved. This problem can be solved to a great extent, if image signal is decomposed properly in different directions, so that low correlation value with the code patterns can be satisfied. We calculate the correlation between the code pattern and the image decomposition coefficients obtained using several DWT and BiDWT. It is observed that the BiDWT provides lower correlation with the code patterns. This is possibly due to the complementary information present in two wavelet systems that offers better directional selectivity compared to classical wavelet transform [5].

\section{PRoposed WATERMARKING TECHNIQUE}

In this section, we present the proposed spread spectrum watermarking technique. The watermarked image $I_{W}$ can be obtained by embedding watermark information $W$ into the image block $I$. The data embedding can be expressed mathematically as follows:

$$
\left[\left(I_{w}\right)_{M}\right]=\left[I_{M}\right]+\alpha \cdot\left[W_{M}\right]
$$

where a set $P$ of $N$ code patterns, each of length $M$, are generated to form watermark sequence $W_{M}$ by performing the following operation.

$$
\left[W_{M}\right]=\sum_{j=1}^{N} b_{j} \cdot\left[P_{M}\right]_{j}
$$

In the above equations, $b_{j} \mathrm{~s}$ represent message bits and $\alpha$ is the gain factor or modulation index.

The watermark embedding strength can be determined from the structure comparison $s(X ; Y)$ [6] using the cross-covariance $\sigma_{x y}$ between the cover (X) and the watermarked image (Y). Data imperceptibility is maintained if the embedding process keeps the change of this functional value to a particular level. In [6], $s(X ; Y)$ in simplified form, is represented as follows:

$$
\sigma_{x y}=s(x, y)=\frac{1}{N-1} \sum_{i=1}^{N}\left(x_{i}-\mu_{x}\right)\left(y_{i}-\mu_{y}\right)
$$

Watermark embedding is additive in nature in code based SS techniques and we denote the amount of data embedding in each coefficient value $\left(x_{i}\right)$ of the cover image $(\mathrm{X})$ by $\Delta x_{i}$. The structure comparison function after data embedding in an image block can be written as follows:

$$
s(x, y)=\frac{1}{N-1}\left[\sum_{i=1}^{N}\left(x_{i}-\mu_{x}\right)^{2}+\sum_{i=1}^{N}\left(x_{i}-\mu_{x}\right)\left(\Delta x-\mu_{\Delta x}\right)\right]
$$

In most cases $\left(\sum_{i=1}^{N}\left|\left(x_{i}-\mu_{x}\right)\right|\right)$ value is greater for high variance image coefficient block than the low variance one. Equations (4) shows that $(\Delta x-$ $\left.\mu_{\Delta x}\right)$ value i.e. watermark strength factor should be smaller for high variance image coefficient block than the low variance one in order to preserve $s(x, y)$ value same in both cases.

The cover image is decomposed in different directions based on the number of messages to be embedded. In the present case two biorthogonal wavelet filters $(6,8)$ and $(4,4)$ are used to decompose image in four different directions and the four different watermark images are embedded in these directions. For each binary valued message bit, pseudo noise (PN) matrix of size identical to the size of the wavelet coefficient matrix is then generated and is modulated by $\mathrm{W}-\mathrm{H}$ matrix. The modulation is done by performing exclusive-OR operation of code pattern with $\mathrm{W}-\mathrm{H}$ matrix. This modulated code pattern $p n_{a}$ is used to embed data in the LL sub band. An orthogonal code pattern 
$p n_{d}$ is obtained by complementing the bits of $p n_{a}$, and is used for data embedding in the $\mathrm{HH}$ subband. If the size of a watermark image is $\left(M_{w} \times M_{w}\right)$, the total number of required code patterns would be $M_{w}{ }^{2}$. The modulation index values for LL \& $\mathrm{HH}$ subbands are taken here 0.7 and 0.3 respectively, although other values (higher for LL subband and lower for $\mathrm{HH}$ subband) may also be considered. To improve robustness in binary modulation, antipodal embedding scheme is used as follows:

$$
X_{l}^{\prime}= \begin{cases}X_{l}+\alpha \cdot P & \text { if } \quad b=0 \\ X_{l}-\alpha \cdot P & \text { if } \quad b=1\end{cases}
$$

where $X_{l}$ represents the wavelet coefficients of the cover image, $X_{l}^{\prime}$ the wavelet coefficients after watermark embedding, $\alpha$ is the modulation index, $P$ is the PN matrix.

To decode message bit, two correlation values (one from LL and other from $\mathrm{HH}$ sub band) are calculated and total $M_{w}{ }^{2}$ (total number of watermark bits) number mean correlation values $\left(\mu_{i}\right)$ are obtained where $i=1,2, . . M_{w}{ }^{2}$. From these mean correlation values, we calculate an overall mean correlation value $(\mathrm{T})$ that is used as the threshold for watermark decoding. The decision rule for the decoded watermark bit is as follows:

if (i) $\mu_{i} \geq T$, the extracted bit is ' 0 ' and

if (ii) $\mu_{i}<T$, the extracted bit is ' 1 '.

To improve capacity further, we use a successive interference cancelation (SIC) scheme [7] where the decision statistics for an embedded bit is obtained by subtracting an estimate of the already detected bits from the received signal as shown in equation (5) below. If estimation is satisfactory, better detection is possible even for smaller modulation index values thus giving rise to more information hiding for a given embedding distortion. This can be explained from the equation given below:

$$
\begin{array}{r}
\tilde{b}_{i, S I C}=\operatorname{sgn}\left(t_{i}\right)=\operatorname{sgn}\left(<P_{i},\left[I+\alpha \cdot \sum_{j=1}^{N} b_{j} \cdot P_{j}\right.\right. \\
\left.\left.-\alpha \cdot \sum_{j=1+1}^{N} \tilde{b}_{j} \cdot P_{j}\right]>(0)\right)
\end{array}
$$

where $t_{i}$ indicates decision variable and $\tilde{b}_{i}$ is the estimate of $b_{i}$.

\section{Performance analysis and eValuation}

We have to look error performance of the proposed scheme. We consider a bit-by-bit hard decoder and assume that the magnitude of interference between the host signal and code pattern is much smaller than the interference due to multiple bit embedding. Fig. 1 shows the conditional density function $f\left(z / y^{k}\right)$ of the detector statistics $z$ along with the region of error (shadded) for binary detection process. The bit error probability can be obtained by integrating the overlapping Gaussian tails:

$$
P_{b}=\frac{1}{2}\left[P\left(\epsilon / Y^{0}\right)+P\left(\epsilon / Y^{1}\right)\right]
$$

where $\epsilon$ indicates for the error. The probability function of the detector statistics, conditioned on a symbol, is the sum of non-Gaussian random variables. If the number of terms is large enough we may use central limit theorem and approximate the sum by a Gaussian distribution with mean $\left(\mu_{i}\right)$ and variance $\left(\sigma_{i}^{2}\right)$ calculated as follows:

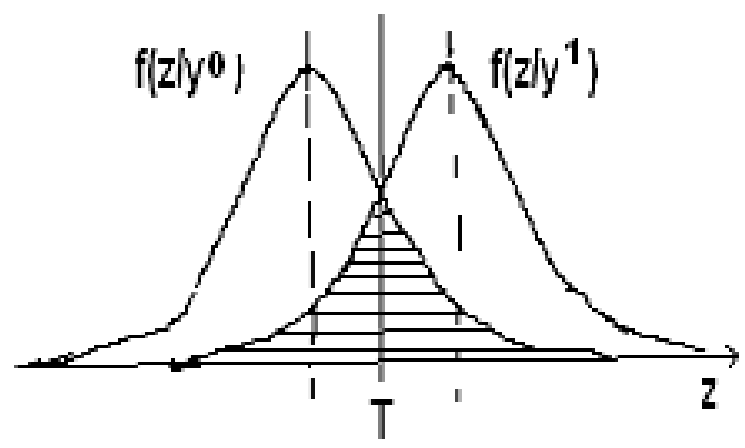

Fig. 1. Conditional pdf for binary decision and error regions

The following equations represent watermarked signal after embedding $N$ bits, decision statistics for the $\mathrm{k}$-th bit, mean and variance of the decision variables respectively:

$$
\begin{gathered}
X_{l}^{\prime}=\sum_{i=1}^{i=N} \sum_{l=1}^{i=L}\left(X_{l}+\alpha \cdot S_{i l}\right) \\
Z_{k}=<X_{l}^{\prime}, S_{k l}>=\sum_{i=1}^{i=N} \sum_{l=1}^{i=L}\left(X_{l}+\alpha \cdot S_{i l}\right) \cdot S_{k l} \\
\mu_{i}=T_{i}=\frac{1}{N} \sum_{i=1}^{i=N} Z_{i} \\
\sigma_{i}^{2}=\operatorname{Var}\left[Z_{k}\right]=E\left[\sum_{i=1}^{i=N} \sum_{l=1}^{i=L}\left(X_{l}+\alpha \cdot S_{i l}\right) \cdot S_{k l}\right]^{2} \\
=\sum_{i=1}^{i=N}\left[\|X\|^{2}+\alpha^{2} . L\right] E\left[S_{k l}^{2}\right]=N\left[\|X\|^{2}+\alpha^{2} \cdot L\right](10)
\end{gathered}
$$


where L represents the number of signal points to be watermarked. In the analysis we consider spreading functions as uniformly distributed random sets of random variable with zero mean and unit variance. The conditional distribution of the detector statistics $f\left(z_{i} / s_{i}\right)$ is given by

$$
f\left(z_{i} / s_{i}\right) \simeq \frac{1}{\sqrt{2 \pi \sigma_{i}^{2}}} e^{-\left(z_{i}-T_{i}\right) / 2 \sigma_{i}^{2}}
$$

We have evaluated the robustness of the proposed technique for various signal processing operations such as linear and nonlinear filtering, dynamic range change, image rescaling, lossy compression (such as JPEG and JPEG 2000), histogram equalization cropping etc over large number of images [8]. The proposed algorithm shows grater resiliency against the stated attacks since each watermark bit is embedded in two different subbands (LL and $\mathrm{HH})$. Redundancy in data embedding offers better stability for the overall mean correlation value $(\mathrm{T})$ and probability of error becomes low even after high degree of signal degradation. Moreover, the robustness performance of BiDWT is much better compared to DWT as the former decomposition offers low correlation with the code patterns and higher energy content for $\mathrm{HH}$ subbands compared to latter decomposition. However, results are not shown due to space limitation. PSNR (peak signal to noise ratio) and MSSIM (mean Structural SIMiliarity) index are used here to quantify invisibility of the hidden data. Table 1 shows how the algorithm improves embedding capacity by embedding multiple binary watermarks in a gray scale image of size $(256 \times 256)$ while data imperceptibility is well maintained. Table 2 compares the performance of the proposed algorithm with our earlier work [4] where $P_{e}$ values indicate BER at quality factor 35 for JPEG 2000 compression operation. The novelty of our algorithm lies in its generalization with respect to the similar type works where host image, watermark and attacks are modeled as familiar Gaussian nature for capacity calculation although the model is far from the actual statistical model of them for practical watermarking system.

We test the performance improvement of detection process using SIC. It is found experimentally that even at quality factor 10 for JPEG 2000 compression, decoding error $\left(P_{b}\right)$ for the first embedded watermark reduces by 0.1 when three other embedded watermarks are removed successively.
TABLE I

IMPERCEPTIBILITY AFTER FOUR $(16 \times 16)$ WATERMARKS

\begin{tabular}{|l|l|l|l|l|}
\hline $\begin{array}{l}\text { Data imper- } \\
\text { ceptibility }\end{array}$ & $\begin{array}{l}\text { single } \\
\text { message }\end{array}$ & $\begin{array}{l}\text { two } \\
\text { messages }\end{array}$ & $\begin{array}{l}\text { three } \\
\text { messages }\end{array}$ & $\begin{array}{l}\text { four } \\
\text { messages }\end{array}$ \\
\hline PSNR(dB) & 35.502 & 35.501 & 34.731 & 34.354 \\
\hline MSSIM & 0.9683 & 0.9681 & 0.9545 & 0.9543 \\
\hline
\end{tabular}

TABLE II

IMPERCEPTIBILITY, CAPACITY AND $P_{e}$ COMPARISON; FIRST THREE ROWS FOR THE PROPOSED ALGO. AND LAST THREE ROWS FOR ALGO. IN [4]

\begin{tabular}{|l|l|l|l|l|}
\hline & $\begin{array}{l}\text { single } \\
\text { message }\end{array}$ & $\begin{array}{l}\text { two } \\
\text { messages }\end{array}$ & $\begin{array}{l}\text { three } \\
\text { messages }\end{array}$ & $\begin{array}{l}\text { four } \\
\text { messages }\end{array}$ \\
\hline PSNR(dB) & 35.502 & 35.501 & 34.731 & 34.354 \\
\hline MSSIM & 0.9683 & 0.9681 & 0.9545 & 0.9543 \\
\hline $\mathrm{P}_{e}$ & 0.0135 & 0.0245 & 0.0298 & 0.0345 \\
\hline PSNR(dB) & 34.23 & 31.56 & 29.90 & 28.45 \\
\hline MSSIM & 0.9523 & 0.9342 & 0.9123 & 0.8942 \\
\hline $\mathrm{P}_{e}$ & 0.0442 & 0.0587 & 0.0745 & 0.1235 \\
\hline
\end{tabular}

\section{CONCLUSiOnS}

A spread spectrum digital image watermarking scheme is proposed for multiple message embedding using suitable biorthogonal wavelet transform. Experiment results show that the embedding capacity can be improved up to three to four times. Also there will be a negligible loss of data imperceptibility and marginal degradation in robustness performance. SIC improves robustness-capacity significantly compared to conventional correlation based approaches.

\section{REFERENCES}

[1] I. J. Cox, J. Kilian, T. Leighton, and T. Shamoon, "Secure spread spectrum watermarking for multimedia," IEEE Trans. on Image Proc., 6:1673-1687, 1997.

[2] J. O. Ruanaidh and T. Pun, "Rotation, scale and translation invariant spread spectrum digital image watermarking,"Signal Proc., 66:303-317,1998.

[3] F. Hartung, J. K. Su, and B. Girod, "Spread spectrum watermarking: Malious attacks and counterattacks," Proc. SPIE, 3657:147-158,1999.

[4] S. P. Maity, M. K. Kundu and T. S. Das, "Design of a robust spread spectrum image watermarking," 4th ICVGIP, PP. 145150, Kolkata, December 2004.

[5] C. S. Burrus, R. A. Gopinath and H. Guo, Introduction to wavelets and wavelet transforms, A Primer, Prentice Hall, Upper Saddle River, NJ, 1997.

[6] Z. Wang, A. C. Bovik, H. R. Sheikh, and E. P. Simoncelli, "Image quality assessment: From error measurement to structural similarity," IEEE Trans. on Image Proc., 13:1-14, 2004.

[7] S. Vedu, Multiuser Detection, Cambridge University Press, 1998.

[8]http://www.cl.cam.ac.uk/fapp2/watermarking 